\title{
Spatially resolved one-dimensional boundary states in graphene-hexagonal boron nitride planar heterostructures
}

Jewook Park ${ }^{1, \star}$, Jaekwang Lee ${ }^{1, \star}$, Lei Liu ${ }^{2}$, Kendal W. Clark ${ }^{3}$, Corentin Durand ${ }^{1}$, Changwon Park ${ }^{1}$, Bobby G. Sumpter ${ }^{1}$, Arthur P. Baddorf ${ }^{1}$, Ali Mohsin², Mina Yoon ${ }^{1}$, Gong Gu² \& An-Ping Li ${ }^{1}$

Two-dimensional interfaces between crystalline materials have been shown to generate unusual interfacial electronic states in complex oxides. Recently, a one-dimensional interface has been realized in hexagonal boron nitride and graphene planar heterostructures, where a polar-on-nonpolar one-dimensional boundary is expected to possess peculiar electronic states associated with edge states of graphene and the polarity of boron nitride. Here we present a combined scanning tunnelling microscopy and first-principles theory study of the graphene-boron nitride boundary to provide a first glimpse into the spatial and energetic distributions of the one-dimensional boundary states down to atomic resolution. The revealed boundary states are about $0.6 \mathrm{eV}$ below or above the Fermi level depending on the termination of the boron nitride at the boundary, and are extended along but localized at the boundary. These results suggest that unconventional physical effects similar to those observed at two-dimensional interfaces can also exist in lower dimensions.

\footnotetext{
${ }^{1}$ Center for Nanophase Materials Sciences, Oak Ridge National Laboratory, Oak Ridge, Tennessee 37831, USA. ${ }^{2}$ The University of Tennessee, Knoxville, Tennessee 37996, USA. ${ }^{3}$ Central Methodist University, Fayette, Missouri 65248, USA. * These authors contributed equally to this work. Correspondence and requests for materials should be addressed to M.Y. (email: myoon@ornl.gov) or to G.G. (email: ggu1@utk.edu) or to A.-P.L. (email: apli@ornl.gov).
} 
$\mathrm{T}$ he ability to control the interfacial properties at the boundary between different materials has formed one of the foundations of modern condensed matter physics and device technology ${ }^{1-5}$. With the advent of graphene and other two-dimensional (2D) crystals, one-dimensional (1D) equivalents of conventional interfaces are envisioned: linear boundaries separating dissimilar materials joint in a single 2D atomic sheet. Graphene and hexagonal boron nitride (hBN) offer a prototypical system to build such a $2 \mathrm{D}$ heterostructure ${ }^{6-11}$. They are isostructural, nearly lattice-matched and isoelectronic, yet their different band structures and the polar-on-nonpolar boundary promise interesting properties arising from their 2D heterostructures ${ }^{12-14}$, just as for interfaces in the threedimensional (3D) counterparts ${ }^{1-5}$. Indeed, the graphene-hBN zigzag boundary is predicted to possess peculiar boundary states $^{12-14}$. Half-metallicity has been reported at this boundary based on theoretical calculations ${ }^{15-19}$, although zigzag graphene nanoribbons have insulating edge states ${ }^{13,20-23}$. Despite the enormous interest in graphene- $\mathrm{hBN}$ heterostructures, the theoretically expected boundary states have not yet been validated experimentally. Some early attempts to address the boundary states have been focused on the connections between graphene and a metallic supporting substrate. For example, when a graphene island is bonded to the edges of a $\mathrm{Pt}(111)$ step, a boundary state is shown to be associated with specific sublattices of the graphene structure ${ }^{24}$. On the other hand, graphene nanoislands grown on $\operatorname{Ir}(111)$ do not show boundary states as the graphene edges are hybridized with the substrate, and the edge states at $\operatorname{Ir}(111)$ are quenched by the strong interaction between the metal and the graphene ${ }^{25}$. Moreover, in a 3D polar-onnonpolar heterostructure of such as $\mathrm{Ge}-\mathrm{GaAs}$ or $\mathrm{LaAlO}_{3}-\mathrm{SrTiO}_{3}$, the polar discontinuity introduces an electric field, which could create an unsustainable electrostatic potential at the interface (termed as the "polar catastrophe ${ }^{26}$ ), leading to electronic or atomic reconstructions $3,5,26,27$ and even preventing an atomically sharp boundary in the latter case $e^{5,27}$. It is unclear whether such a polar catastrophe would occur in a 2D polar-on-nonpolar heterostructure of graphene-hBN.
Here we combine scanning tunnelling microscopy (STM) and first-principles theory methods to study the spatial and energetic distributions of the electronic boundary states in the graphenehBN in-plane heterostructures. The interfaces studied here are crystallographically coherent with sharp transitions from graphene zigzag edges to $\mathrm{B}$ (or $\mathrm{N}$ )-terminated monolayer $\mathrm{hBN}$ on a $\mathrm{Cu}$ foil substrate ${ }^{6}$. The boundary states are revealed down to the atomic level with energy levels about $0.6 \mathrm{eV}$ below and above the Fermi level, respectively, for the B- and N-terminated boundaries. The boundary states are shown to be extended along the boundary, and exponentially decay into the bulk of graphene and $\mathrm{hBN}$ with decay lengths in a range of $0.24-0.51 \mathrm{~nm}$. The boundary states come from the mixing of $\pi$ orbitals of $\mathrm{C}, \mathrm{B}$ and $\mathrm{N}$ at each edge, with the occupied bonding states $\left(\pi_{\mathrm{CB}}\right)$ at B-terminated edges and the unoccupied anti-bonding states $\left(\pi^{*} \mathrm{CN}\right)$ at $\mathrm{N}$-terminated edges. The effect of the electric field induced by the polarity discontinuity at the interface is discussed, which can be effectively screened out by the charge carriers from graphene and $\mathrm{Cu}$ substrate.

\section{Results}

STM topography of graphene- $h B N$ heterostructures. An inplane, single-atomic layer graphene- $\mathrm{hBN}$ heterostructure grown on a $\mathrm{Cu}$ foil ${ }^{6}$ is used to experimentally investigate the boundary states in this work owing to the weak substrate interaction ${ }^{28}$ and a sharp boundary at the atomic scale ${ }^{6}$. Figure $1 \mathrm{~b}$ shows an STM image of two adjacent graphene-hBN junctions formed by an hBN region between two graphene regions, with magnified images of the respective boundaries shown in Fig. 1a and Fig. 1c. The zigzag boundary orientations are indicated in Fig. 1a,c. The image contrast in Fig. 1a is dominated by a substrate step, which appears to be close to an armchair orientation. However, superimposing the schematic atomic lattice on the image and comparing with $\mathrm{d} I / \mathrm{d} V$ map (details shown in Supplementary Fig. 1) reveal that the structures of the boundary are actually zigzag. The simultaneously acquired scanning tunnelling spectroscopy (STS) map (Fig. 1d) shows distinctively different
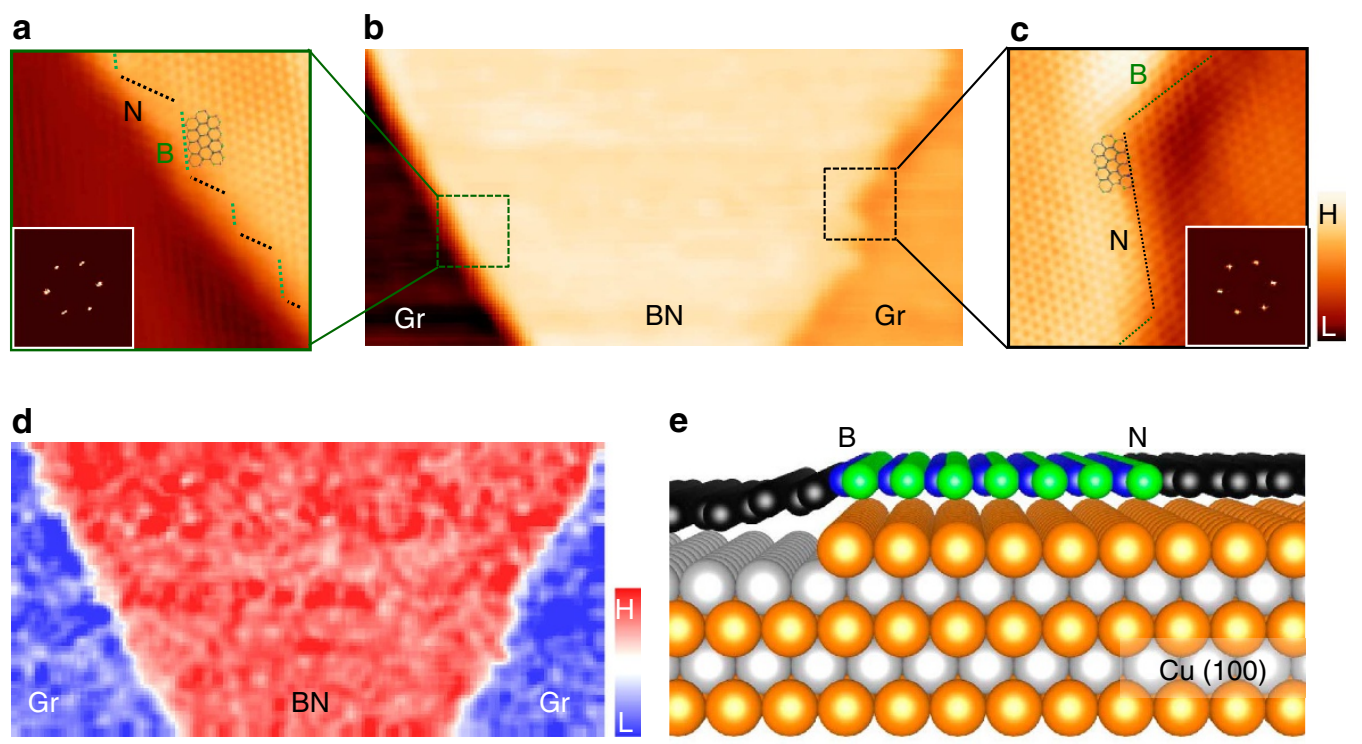

Figure 1 | STM topography of a planar graphene-hBN-graphene heterostructure. (a-c) STM images, with large-scale image in b showing two adjacent boundaries $\left(50 \times 25 \mathrm{~nm}^{2}, 0.5 \mathrm{~V}, 200 \mathrm{pA}\right)$, and $\mathbf{a}$ and $\mathbf{c}$ showing atomically resolved images near each boundary $\left(6 \times 6 \mathrm{~nm}^{2}\right)$. The edge terminations of hBN are marked with dotted lines. Insets: fast Fourier transform from the images. (d) Simultaneously obtained dl/dV map with image in $\mathbf{b}$ (AC modulation $500 \mathrm{~Hz}, 50 \mathrm{mV}$ ). (e) Schematic illustration of two boundaries on $\mathrm{Cu}(100)$ substrate projected along <011> direction. The height difference of the two junctions is due to an underlying $\mathrm{Cu}(100)$ step $(\Delta h=1.8 \AA$ ). ' $\mathrm{B}$ ' and ' $\mathrm{N}$ ' indicate the hBN region termination, determined by STS in conjunction with firstprinciples calculations. $\mathrm{Gr}$ and $\mathrm{BN}$ represent graphene and $\mathrm{hBN}$ regions for simplicity. 
contrasts between graphene (blue) and hBN (red) regions. Our assignments of graphene and hBN regions are based on STS measured across the boundaries (Supplementary Figs 2 and 3), where graphene and hBN show distinctively different characteristics with graphene showing a dip at Dirac point of $-0.35 \mathrm{~V}$ and the $\mathrm{hBN}$ showing a large band gap of $\sim 4 \mathrm{eV}$ (refs 6,29-32). The boundary on the right side (Fig. 1c) shows a small height difference $(\sim 0.7 \AA)$ originating from the difference in the electronic density of states between the graphene and $\mathrm{hBN}$, and the left boundary (Fig. 1a) has a height difference of $2.5 \AA$ that consists of a substrate step $(1.8 \AA)$ on $\mathrm{Cu}(100)$, the predominant surface orientation of the $\mathrm{Cu}$ foil substrate ${ }^{6,29}$ (Supplementary Fig. 4). Figure 1e depicts a schematic of these two boundaries on the substrate.

The boundary structures are further confirmed by simultaneously acquired atomic-resolution STM images and STS maps. As shown in Supplementary Fig. 2, all the graphene-hBN boundaries are found to contain some segments oriented $120^{\circ}$ away from the primary edge direction, which have different terminations than the primary edges, considering the lattice symmetry of $\mathrm{hBN}$ and that a zigzag interface is more energetically favourable (about $1.37 \mathrm{eV}$ per edge carbon atom) than a Kleintype interface ${ }^{33}$ for this graphene-hBN heterostructure on a $\mathrm{Cu}$ substrate. By comparing measured boundary states with theoretical analysis (discussed later), we identify boundary terminations as indicated in Fig. 1a,c.

Energetic distributions of boundary states. We now examine the electronic properties of both $\mathrm{B}$ - and $\mathrm{N}$-terminated graphene$\mathrm{hBN}$ boundaries by measuring the differential conductance $\mathrm{d} I / \mathrm{d} V$, which is proportional to the local electronic density of states (LDOS) distribution, especially at low bias voltages. Figure 2a shows the $\mathrm{d} I / \mathrm{d} V$ map at a bias voltage of $-0.6 \mathrm{~V}$ for the two boundaries displayed in Fig. 1b. The electronic states can be seen at both boundaries as intense red-coloured regions. Enhanced electronic states are observed only at the B-terminated boundaries (indicated by green dotted lines), not at the N-terminated boundary (indicated by black dotted lines) at this bias voltage. The measured $\mathrm{d} I / \mathrm{d} V$ curves in Fig. $2 \mathrm{~b}-\mathrm{d}$ show LDOS variations along three different lines going from graphene to $h B N$ as indicated in Fig. 2a. Remarkably, the $\mathrm{d} I / \mathrm{d} V$ curves at the B-terminated boundaries (red curves) display enhanced LDOS at around $-0.6 \mathrm{~V}$ that corresponds to the intense red-coloured region in the $\mathrm{d} I / \mathrm{d} V$ map (Fig. 2a). On the other hand, the $\mathrm{N}$-terminated boundary (Fig. 2d) does not show an increase in LDOS at $-0.6 \mathrm{~V}$; instead, it shows highly localized electronic states at positive bias as explained below.

Spatial distributions of boundary states. The spatial confinement of boundary states can be visualized clearly by plotting the measured $\mathrm{d} I / \mathrm{d} V$ intensity contours at a constant bias voltage. Figure $3 \mathrm{~b}$ shows the atomically resolved spatial distributions of measured $\mathrm{d} I / \mathrm{d} V$ across a graphene-hBN boundary, acquired simultaneously with the STM image (Fig. 3a) at $0.6 \mathrm{~V}$. At this bias, the LDOS enhancement appears only at $\mathrm{N}$-terminated edges, and is confined within a few atomic lattices near the boundary. Moreover, the $\mathrm{d} I / \mathrm{d} V$ intensity at constant bias decays exponentially from the boundary into the graphene and hBN regions (Fig. 3c). We find that the boundary state decays slightly faster in $\mathrm{hBN}$ than in graphene, with a larger decay length on the graphene side $(0.47 \pm 0.08 \mathrm{~nm})$ than the $\mathrm{hBN}$ side $(0.32 \pm 0.05 \mathrm{~nm})$. In contrast, the B-terminated boundary states become more pronounced at $-0.6 \mathrm{~V}$ as shown in Fig. $3 \mathrm{~d}-\mathrm{f}$ with decay lengths of $0.51 \pm 0.17$ and $0.24 \pm 0.05 \mathrm{~nm}$ on the graphene and the hBN side, respectively.

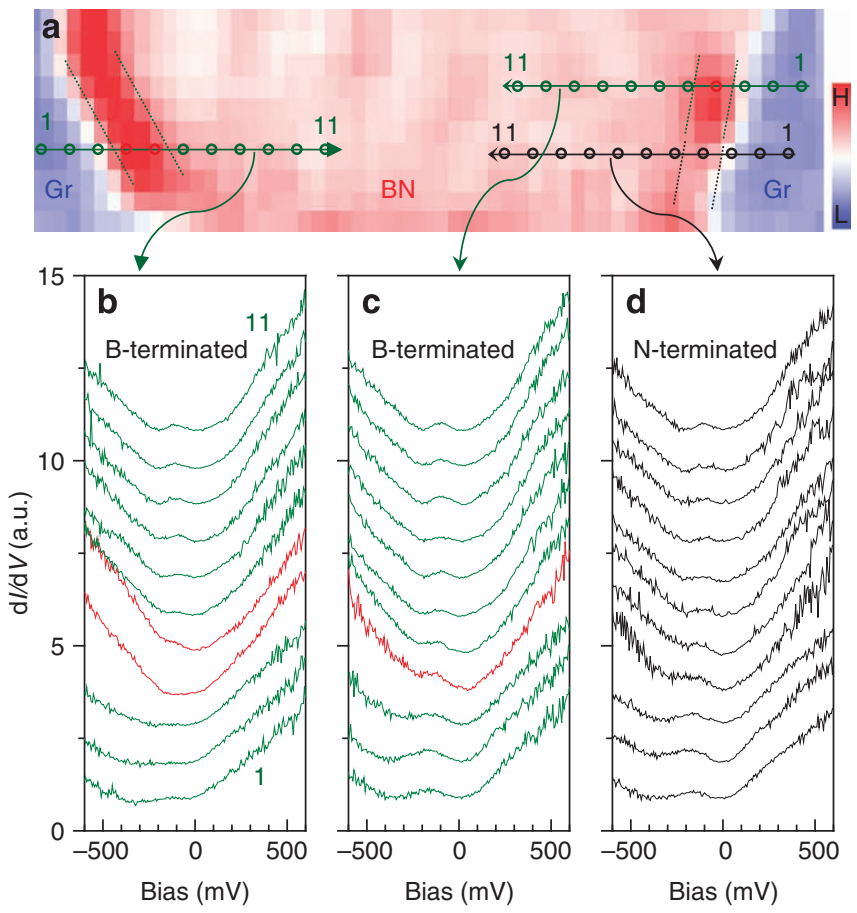

Figure 2 | Electronic boundary states measured by STS. (a) $d / / d V$ conductance map $\left(45 \times 12.5 \mathrm{~nm}^{2}\right)$ of the $\mathrm{Gr}-\mathrm{hBN}-\mathrm{Gr}$ regions measured at $-0.6 \mathrm{~V}$ to the sample. The boundary states (between green dotted lines) are noticeable as intense red colour. Circles on arrowed lines mark positions of STS from graphene to the hBN, where green lines go across the $\mathrm{B}$-terminated regions and black lines across the $\mathrm{N}$-terminated region. (b-d) $\mathrm{d} l / \mathrm{d} V$ curves acquired along three lines marked in $\mathbf{a}$. The red curves correspond to those acquired between green dotted lines in $\mathbf{a}$. Spectroscopy data are offset for clarity.

These boundary states are reproducibly observed in an energy range of $0.45-0.78 \mathrm{eV}$ below the Fermi level for the B-terminated boundaries and $0.60-0.90 \mathrm{eV}$ above the Fermi level for the $\mathrm{N}$-terminated boundaries (see Supplementary Figs 2,5,6 for more details), confirming that the appearance of these states are not experimental artefacts but an intrinsic boundary property. Moreover, all the boundary states are localized at and extended along the boundary, although the electronic states at B- and N-terminated boundaries have clearly different energy levels.

First-principles calculations of boundary states. We now compare the experimental observations with first-principles density functional theory (DFT) calculations (see Methods for the details). As illustrated in Fig. 4a, we consider a supercell in which an hBN ribbon of 7 zigzag rows joint by a 12-row graphene ribbon, on a 5-layer $\mathrm{Cu}$ slab, oriented (100) with a $0^{\circ}$ rotation angle. In view of the experimentally identified superstructure (six graphene or $\mathrm{hBN}$ unit cells match to five $\mathrm{Cu}(100)$ surface lattice unit cells in the direction perpendicular to the boundary ${ }^{6}$ ), graphene-hBN supercell is not unique. We consider a flat graphene-hBN on $\mathrm{Cu}(100)$ while excluding local lattice distortions that vary with supercell configurations. We project the density of states on each hBN or graphene zigzag row in the unit cell (see Supplementary Fig. 7). To facilitate the comparison of the calculated LDOS with the measured $\mathrm{d} I / \mathrm{d} V$, we plot the LDOS contour within a small energy region (from $-0.8 \mathrm{eV}$ to $0.4 \mathrm{eV}$ ) near the Fermi level in Fig. $4 \mathrm{~b}, \mathrm{c}$ for the B- and $\mathrm{N}$-terminated boundaries, respectively, with the contribution 

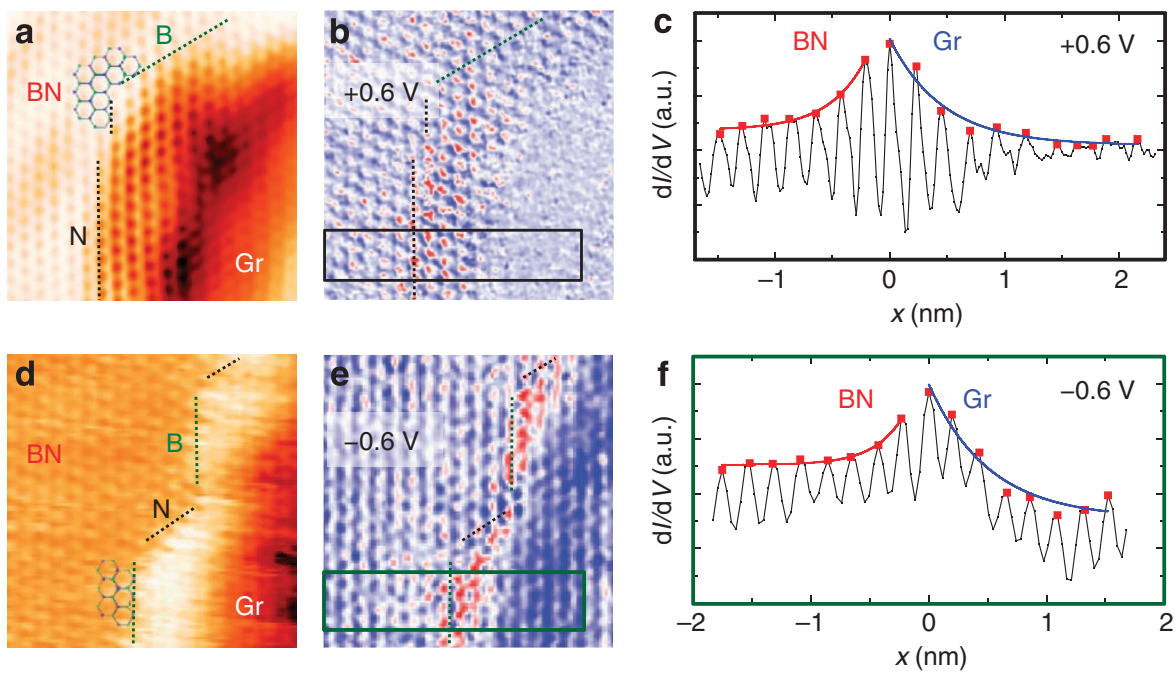

Figure 3 | Spatial distributions of boundary states. (a,d) Atomically resolved topography image of planar junction showing the seamless connections between graphene (right) and hBN (left) regions. (b,e) Simultaneously obtained dl/dV conductance map with the images (a,d) displaying distinct boundary states in red (with the same colour scheme as shown in Fig. 2a) at different bias voltages with the same set point (200 pA). Image sizes: a and $\mathbf{b}$, $4.2 \times 4.2 \mathrm{~nm}^{2} ; \mathbf{d}$ and $\mathbf{e}, 4 \times 4 \mathrm{~nm}^{2}$. Hexagonal lattice of hBN is overlaid on topography. Green and black dotted lines indicate B- and N-terminated boundaries, respectively. (c,f) Averaged line profiles across the boundary regions (marked with boxes in $\mathbf{b}$ and $\mathbf{e}$ ) showing exponential decay of the interfacial state into the both graphene and hBN regions. Red and blue lines are exponential fitting curves.

a

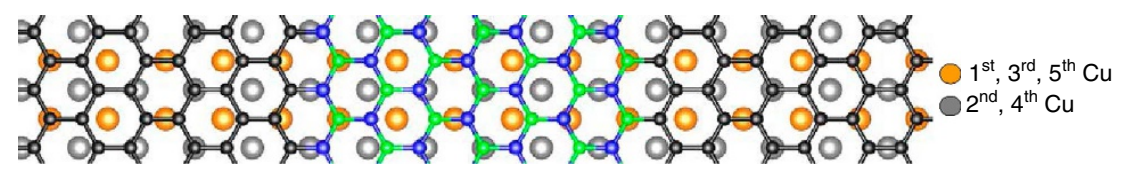

b
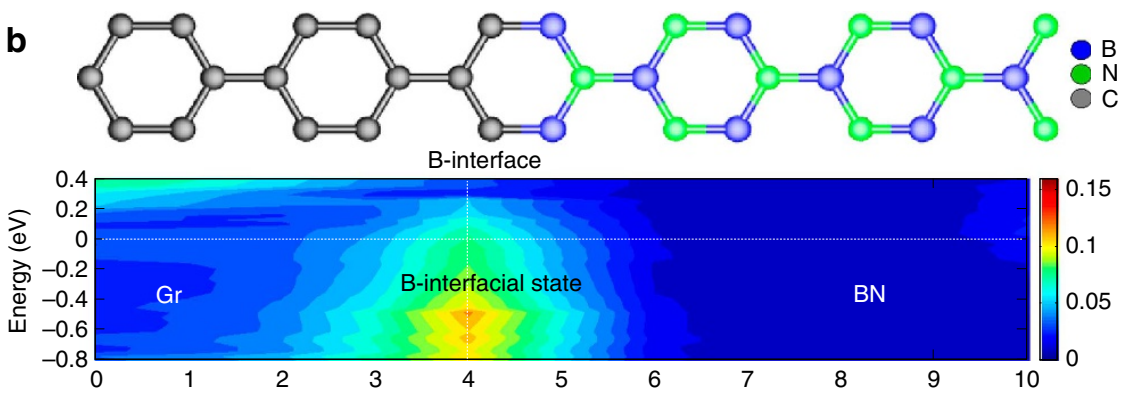

C
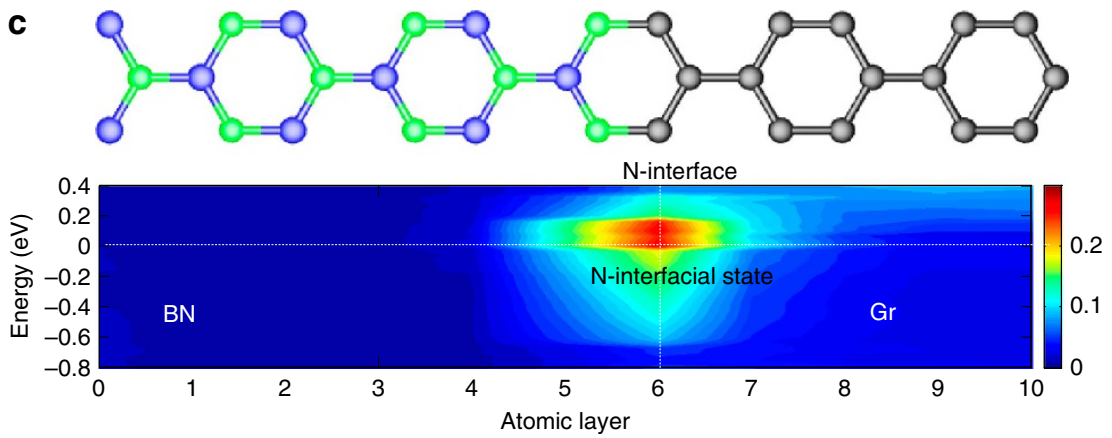

Figure 4 | Calculated boundary states of the planar graphene-hBN-graphene heterostructure on a Cu(100) substrate. (a) Schematic of the heterostructure (top view). Seven hBN zigzag rows are laterally sandwiched by 12 graphene rows. Five Cu layers are considered as the substrate. B atoms are specified in blue, $\mathrm{N}$ atoms in green and carbon atoms in black. (b) Contour map related to the B-terminated state made up of LDOS projected onto 11 zigzag rows (5 graphene and $6 \mathrm{hBN}$ rows) across the B-terminated boundary. B-terminated states are located around $0.6 \mathrm{eV}$ below the Fermi level.

(c) Contour map related to the $\mathrm{N}$-terminated state across the $\mathrm{N}$-terminated boundary. The boundary states are located around $0.1 \mathrm{eV}$ above the Fermi level.

from $\mathrm{Cu}$ excluded for clarity. For the B-terminated boundary (Fig. 4a), the boundary states are located about $0.6 \mathrm{eV}$ below the Fermi level and localized within only a few rows (row-to-row distance is $2.165 \AA$, given a $1.44-\AA \mathrm{C}-\mathrm{C}$ or $\mathrm{B}-\mathrm{N}$ length) of the interface. The spatial distribution of LDOS extends into the graphene region more deeply than into the $\mathrm{hBN}$ region, with the decay length in graphene $3.1 \AA$ and in hBN $2.0 \AA$, respectively. The highest intensity of LDOS appears at the $\mathrm{C}$ site for both 
B- and N-terminated boundary states, consistent with the measured $\mathrm{d} I / \mathrm{d} V$ (Fig. 3c,f). Both the calculated energy position of the B-terminated boundary state and its decay length are in good agreement with the STS measurement.

The calculations employing the DFT exchange and correlation (XC) functional of the local density approximation (LDA) also yield highly localized states at the N-terminated boundary located around $0.1 \mathrm{eV}$ above the Fermi level (Fig. 4c), lower than that measured by STS mapping. As LDA calculations usually underestimate the band gaps and especially the energies of unoccupied states, we further performed Heyd - Scuseria - Ernzerhof (HSE) hybrid functional calculations ${ }^{34}$ on a small supercell (see Methods for details) to account for the discrepancy in the $\mathrm{N}$-terminated boundary state level. It is found that the energy level of B-terminated states remain unchanged from the LDA result on the same supercell, but the $\mathrm{N}$-terminated states move up to $\sim 0.6 \mathrm{eV}$ above the Fermi level (see Supplementary Fig. 8); and the shift accounts for the difference in the $\mathrm{N}$-terminated boundary states between the experiment and the LDA results in Fig. 4c.

Although the interactions with the $\mathrm{Cu}$ substrate are very weak $^{28}$, we find that there are still hybridizations between the $d$-orbitals of $\mathrm{Cu}$ and the $\pi$-orbitals of graphene and hBN near the B-terminated boundary (see Supplementary Fig. 9). However, no appreciable interaction is seen between N-terminated states and $\mathrm{Cu}$ states. These dissimilar interactions arise from the different electronegativities of $\mathrm{B}$ and $\mathrm{N}$. As a comparison, we have compared the calculated LDOS with and without a $\mathrm{Cu}$ substrate (Supplementary Fig. 10) and found that the N-terminated boundary states remain largely unchanged, but the $\mathrm{B}$-terminated states are modified by the $\mathrm{Cu}$ substrate due to the hybridization with $\mathrm{Cu}$ substrate.

In comparison, we carried out DFT calculations on an in-plane graphene-hBN junction on $\mathrm{Cu}(111)$. As shown in Supplementary Fig. 11, the calculated results for both B- and N-terminated boundaries are essentially the same as those on $\mathrm{Cu}(100)$, confirming that crystallographic orientation of $\mathrm{Cu}$ has little effect on the boundary states.

\section{Discussion}

We now clarify the nature of the observed boundary states on the basis of the combined experimental and theoretical study. When two semi-infinite sheets of graphene and $\mathrm{hBN}$ form a zigzag heterojunction on a $\mathrm{Cu}$ substrate, the mixing of $\pi$-orbitals of $\mathrm{C}, \mathrm{B}$ and $\mathrm{N}$ at each edge gives rise to four sets of bands that correspond to the bonding and anti-bonding states between $\mathrm{C}-\mathrm{N}$ and $\mathrm{C}-\mathrm{B}$ (Supplementary Fig. 12). Although the boundary states are spin polarized in the free-standing case ${ }^{13}$ as are the edge states of freestanding zigzag hBN nanoribbons ${ }^{35}$, calculations by us and others ${ }^{36}$ indicate that the boundary magnetism disappears upon adsorption onto a $\mathrm{Cu}$ substrate. The relevant bands, close to the Fermi level, are the occupied bonding states $\left(\pi_{\mathrm{CB}}\right)$ at B-terminated edges and the unoccupied anti-bonding states $\left(\pi^{*} \mathrm{CN}\right)$ at $\mathrm{N}$-terminated edges, both localized at the interface. The localized states lay $\sim 0.6 \mathrm{eV}$ below and above the Fermi level, respectively (Fig. 3).

In addition, different electronegativities of $\mathrm{B}$ and $\mathrm{N}$ make the $\mathrm{hBN}$ strip the $2 \mathrm{D}$ equivalent to a polar slab. We calculated that a free-standing eight-row hBN strip experiences a polar field of $\sim 0.11 \mathrm{eV} \AA^{-1}$, which is reduced to about $0.06 \mathrm{eV}^{-1}$ by screening when joint with graphene. The $\mathrm{Cu}$ substrate brings about an additional screening, as corroborated in our calculations. In a 3D polar-on-nonpolar heterostructure, 2D charge sheets create a constant overall electric field and the electrostatic potential would diverge with increasing thickness, leading to a 'polar catastrophe'26. The polar catastrophe can be averted by charge reconstruction ${ }^{26}$ or atom rearrangement ${ }^{5}$. For (100) or (111) Ge-GaAs interfaces, which are the higher-dimensional analogies of the zigzag graphene-hBN boundaries, atom rearrangement prevents an atomically sharp interface ${ }^{5,27}$. In a 2D polar-on-nonpolar heterostructure, the electric field induced by the polar discontinuity at the boundary is inversely proportional to the distance, and the electrostatic potential would also diverge although not as fast as in 3D. Furthermore, the charge carriers from graphene and $\mathrm{Cu}$ substrate can effectively screen out the polar field. As such, the polarity discontinuity in 2D may not be as catastrophic. Nevertheless, planar graphene$\mathrm{hBN}$ heterostructures provide an excellent platform for exploring novel physics in a $1 \mathrm{D}$ system-the interface in $2 \mathrm{D}$ space.

\section{Methods}

Sample preparation and STM/STS experiments. The graphene-hBN planar junction on $\mathrm{Cu}$ foil sample was prepared using the $2 \mathrm{D}$ heteroepitaxial growth technique $^{6}$. Moreover, (100) has been found to be the predominant $\mathrm{Cu}$ surface orientation as confirmed by low-energy electron microscopy and low-energy electron diffraction ${ }^{6}$ and X-ray diffraction and electron backscatter diffraction (Supplementary Fig. 4). STM/STS were performed using a variable-temperature STM (Omicron) with Nanonis (SPECS) controller and an electrochemically etched tungsten tip. All measurements were carried out in ultra-high vacuum $\left(<3 \times 10^{-10}\right.$ Torr $)$ at room temperature. We annealed the sample at $430^{\circ} \mathrm{C}$ for $48 \mathrm{~h}$ for cleaning. We used constant current mode for topography images and a lock-in technique for differential conductance measurement with $500 \mathrm{~Hz}$, $40-100 \mathrm{mV}$ AC modulations. All STM images and spectroscopy data were analysed and processed with WSxM software.

First-principles calculations. First-principles calculations, based on DFT, were performed using the Vienna Ab initio Simulation Package. The projectoraugmented wave method was used to mimic the ionic cores, while the LDA considering the Ceperly-Alder-Perdew and Zunger (CA-PZ) functional was employed for the XC functional. In addition, an HSE-screened hybrid XC functional ${ }^{34}$ was used to more properly determine the energies of interface states. The overall fraction of Fock exchange and the length scale $\omega^{-1}$ for exchange screening are adopted as the HSE12s (ref. 37) form of $a=0.425$ and $\omega=0.408 \AA^{-1}$ Atomic positions, as well as lattice parameters, were optimized using a conjugate gradient algorithm. The ionic and electronic relaxations were performed by applying a convergence criterion of $5 \times 10^{-2} \mathrm{eV} \AA^{-1}$ per ion and $10^{-4} \mathrm{eV}$ per electronic step, respectively. The rectangular graphene-BN hybrid structure, $43.3 \times 2.5 \AA$, is used for the LDOS calculations, and a vacuum of $16 \AA$ between the hybrids is considered. Also, $1 \times 10 \times 1$ Monkhorst-Pack meshes were used to perform the integration over the Brillouin zone.

\section{References}

1. Bert, J. A. et al. Direct imaging of the coexistence of ferromagnetism and superconductivity at the $\mathrm{LaAlO}_{3} / \mathrm{SrTiO}_{3}$ interface. Nat. Phys. 7, 767-771 (2011).

2. Li, L., Richter, C., Mannhart, J. \& Ashoori, R. C. Coexistence of magnetic order and two-dimensional superconductivity at $\mathrm{LaAlO}_{3} / \mathrm{SrTiO}_{3}$ interfaces. Nat. Phys. 7, 762-766 (2011).

3. Ohtomo, A. \& Hwang, H. Y. A high-mobility electron gas at the $\mathrm{LaAlO}_{3} /$ $\mathrm{SrTiO}_{3}$ heterointerface. Nature 427, 423-426 (2004).

4. Reyren, N. et al. Superconducting interfaces between insulating oxides. Science 317, 1196-1199 (2007)

5. Kroemer, H. Nobel Lecture: Quasielectric fields and band offsets: teaching electrons new tricks. Rev. Mod. Phys. 73, 783-793 (2001).

6. Liu, L. et al. Heteroepitaxial growth of two-dimensional hexagonal boron nitride templated by graphene edges. Science 343, 163-167 (2014).

7. Han, G. H. et al. Continuous growth of hexagonal graphene and boron nitride in-plane heterostructures by atmospheric pressure chemical vapor deposition. ACS Nano 7, 10129-10138 (2013).

8. Sutter, P., Cortes, R., Lahiri, J. \& Sutter, E. Interface formation in monolayer graphene-boron nitride heterostructures. Nano Lett. 12, 4869-4874 (2012).

9. Ci, L. et al. Atomic layers of hybridized boron nitride and graphene domains. Nat. Mater. 9, 430-435 (2010).

10. Gao, Y. et al. Toward single-layer uniform hexagonal boron nitride-graphene patchworks with zigzag linking edges. Nano Lett. 13, 3439-3443 (2013).

11. Kim, S. M. et al. Synthesis of patched or stacked graphene and hBN flakes: a route to hybrid structure discovery. Nano Lett. 13, 933-941 (2013).

12. Okada, S., Igami, M., Nakada, K. \& Oshiyama, A. Border states in heterosheets with hexagonal symmetry. Phys. Rev. B 62, 9896-9899 (2000). 
13. Pruneda, J. M. Origin of half-semimetallicity induced at interfaces of C-BN heterostructures. Phys. Rev. B 81, 161409 (2010).

14. Liu, Y., Bhowmick, S. \& Yakobson, B. I. BN white graphene with 'colorful' edges: the energies and morphology. Nano Lett. 11, 3113-3116 (2011).

15. Bhowmick, S., Singh, A. K. \& Yakobson, B. I. Quantum dots and nanoroads of graphene embedded in hexagonal boron nitride. J. Phys. Chem. C 115, 9889-9893 (2011)

16. Liu, Y., Wu, X., Zhao, Y., Zeng, X. C. \& Yang, J. Half-metallicity in hybrid graphene/boron nitride nanoribbons with dihydrogenated edges. J. Phys. Chem. C 115, 9442-9450 (2011).

17. Xiao, H. P. et al. Size effect of half-metallic properties of BN/C hybrid nanoribbons. Phys. B: Cond. Matt. 407, 4770-4772 (2012).

18. Bristowe, N. C., Stengel, M., Littlewood, P. B., Artacho, E. \& Pruneda, J. M. One-dimensional half-metallic interfaces of two-dimensional honeycomb insulators. Phys. Rev. B 88, 161411 (2013).

19. Dutta, S., Manna, A. K. \& Pati, S. K. Intrinsic half-metallicity in modified graphene nanoribbons. Phys. Rev. Lett. 102, 096601 (2009).

20. Castro Neto, A. H., Guinea, F., Peres, N. M. R., Novoselov, K. S. \& Geim, A. K. The electronic properties of graphene. Rev. Mod. Phys. 81, 109-162 (2009).

21. Son, Y.-W., Cohen, M. L. \& Louie, S. G. Half-metallic graphene nanoribbons. Nature 444, 347-349 (2006).

22. Ritter, K. A. \& Lyding, J. W. The influence of edge structure on the electronic properties of graphene quantum dots and nanoribbons. Nat. Mater. 8, 235-242 (2009).

23. Tao, C. et al. Spatially resolving edge states of chiral graphene nanoribbons. Nat. Phys. 7, 616-620 (2011).

24. Merino, P. et al. Sublattice localized electronic states in atomically resolved graphene-Pt(111) edge-boundaries. ACS Nano 8, 3590-3596 (2014).

25. Li, Y. et al. Absence of edge states in covalently bonded zigzag edges of graphene on $\operatorname{Ir}(111)$. Adv. Mater. 25, 1967-1972 (2013).

26. Nakagawa, N., Hwang, H. Y. \& Muller, D. A. Why some interfaces cannot be sharp. Nat. Mater. 5, 204-209 (2006).

27. Harrison, W. A., Kraut, E. A., Waldrop, J. R. \& Grant, R. W. Polar heterojunction interfaces. Phys. Rev. B 18, 4402-4410 (1978)

28. Khomyakov, P. A. et al. First-principles study of the interaction and charge transfer between graphene and metals. Phys. Rev. B 79, 195425 (2009).

29. Tian, J. et al. Graphene induced surface reconstruction of Cu. Nano Lett. 12 , 3893-3899 (2012)

30. Gao, L., Guest, J. R. \& Guisinger, N. P. Epitaxial graphene on $\mathrm{Cu}(111)$. Nano Lett. 10, 3512-3516 (2010).

31. Hwang, B. et al. Electron-beam assisted growth of hexagonal boron-nitride layer. Curr. Appl. Phys. 13, 1365-1369 (2013).

32. Joshi, S. et al. Boron nitride on $\mathrm{Cu}(111)$ : an electronically corrugated monolayer. Nano Lett. 12, 5821-5828 (2012).
33. Klein, D. J. Graphitic polymer strips with edge states. Chem. Phys. Lett. 217, 261-265 (1994).

34. Heyd, J., Scuseria, G. E. \& Ernzerhof, M. Hybrid functionals based on a screened Coulomb potential. J. Chem. Phys. 118, 8207-8215 (2003).

35. Zheng, F. et al. Half metallicity along the edge of zigzag boron nitride nanoribbons. Phys. Rev. B 78, 205415 (2008).

36. Li, Y. \& Mazzarello, R. Structural and electronic properties of hybrid graphene and boron nitride nanostructures on Cu. Phys. Rev. B 88, 045317 (2013).

37. Moussa, J. E., Schultz, P. A. \& Chelikowsky, J. R. Analysis of the Heyd-ScuseriaErnzerhof density functional parameter space. J. Chem. Phys. 136, 204117 (2012).

\section{Acknowledgements}

This research was conducted at the Center for Nanophase Materials Sciences, which is sponsored at Oak Ridge National Laboratory by the Scientific User Facilities Division, Office of Basic Energy Sciences, US Department of Energy (DOE). A portion of theory work (J.L.) is supported by the Laboratory Directed Research and Development Program of Oak Ridge National Laboratory, managed by UT-Battelle, LLC, for the US DOE. This research used resources of the National Energy Research Scientific Computing Center, a DOE Office of Science User Facility supported by the Office of Science of the US DOE under contract no. DE-AC02-05CH11231. The work at UTK was supported by NSF (ECCS-1231808) and DARPA (approved for public release; distribution is unlimited).

\section{Author contributions}

J.P. and J.L. contributed equally to this work. A.-P.L. conceived the project and designed the STM experiments and M.Y. designed the theory tasks. Measurements were carried out by J.P., K.W.C, C.D. and A.-P.L.; samples were prepared by L.L. and G.G.; and the first-principles calculations were performed by J.L., C.P. and M.Y. All authors participated in the data interpretation and paper writing

\section{Additional information}

Supplementary Information accompanies this paper at http://www.nature.com/ naturecommunications

Competing financial interests: The authors declare no competing financial interests.

Reprints and permission information is available online at http://npg.nature.com/ reprintsandpermissions/

How to cite this article: Park, J. et al. Spatially resolved one-dimensional boundary states in graphene-hexagonal boron nitride planar heterostructures. Nat. Commun. 5:5403 doi: 10.1038/ncomms6403 (2014) 